\title{
Timing of the Electroretinogram Response and Dark Adaptation
}

\author{
JEAN REAL BRUNETTE AND GILLES LAFOND
}

SUMMARY: The time response of the Electroretinogram (ERG) is an essential part of the examination. Peak time of response increases with dark adaptation and decreases with the intensity of stimulation. When working with constant amplitude during dark adaptation sensitivity increases but the intensity of stimulation decreases. It is essential to clarify the relationship of these two factors for valid interpretation of results. The present work suggests that the state of adaptation or retinal sensitivity affects amplitude, while peak time of the response is modified by the absolute value of the stimulating source whatever the state of adaptation. This observation has been done on rod responses and is valid as long as pigments remain unbleached and able to respond.

RÉSUMÉ: L'évaluation du paramètre temps de la réponse ERG clinique est essentielle à l'interprétation et la classification des pathologies. Or le temps de culmination est directement proportionnel au niveau d'adaptation à l'obscurité et inversement à l'intensité de stimulation. Au cours du relevé des réponses au cours de l'adaptation à l'obscurité, la sensibilité augmente mais l'intensité de stimulation décroit si on travaille à amplitude constante. Il devient donc essentiel de clarifier les interrelations entre ces 2 facteurs. Le présent travail suggére que l'état d'adaptation, ou sensibilité rétinienne, affecte l'amplitude, ou le seuil, et que cet état d'adaptation peut ainsi être évalué. Le temps de réponse par contre est fonction de la valeur absolue de la source lumineuse stimulante, indépendamment du niveau d'adaptation tant que demeurent des pigments non blanchis.

From the Laboratoire de Physiologie Visuelle, Department of Ophthalmology, Université de Montreal, Montréal, Canada. This study was supported by the Canadian Medical Research Council, Grant No. MA-2593.

Reprint requests to Dr. J. R. Brunette, c/o Dept. of Ophthaimology, Centre Hospitalier Universitaire, De Sherbrooke, Sherbrooke, Quebec JIH SN4 Canada.

\section{INTRODUCTION}

The time parameter of the electroretinogram (ERG) response has evolved as an important element in the evaluation of the response. The synchronising of all firing receptors, advocated by Berson et al. (1969) and exemplified by Ganzfeld stimulation, has allowed the evaluation of this parameter. It appears that peak time varies with different pathology and independently of amplitude. Working with constant amplitude has emphasized these observations (Brunette, 1973). The time response may vary before, and independently of, amplitude changes.

However, the time response has been shown to increase during the course of dark adaptation when, as in threshold ERG, constant amplitudes are maintained throughout the dark adaptation (Brunette and Lafond, 1977). On the other hand, when the intensity of the stimulation is increased, peak time value decreases (Brunette and Lafond, 1977). The question arises whether peak times increased during constant amplitude ERG dark adaptation were due to dark adaptation or to the consequent decrease of the stimulus intensity by gain of sensitivity, since the time response is influenced by both factors.

The present work attempts to separate the effects of adaptation from stimulation intensity on the time response. This problem is crucial in the interpretation of clinical ERGs. This is particularly so with elevated thresholds (decreased sensitivity), since to obtain a threshold response intensity of stimulation must be increased.

MATERIAL AND METHODS

Experiments were done using normal, healthy, young adult Rhesus Monkeys. Previous to all experiments, subjects were light preadapted to a constant diffuse background. In the first series of experiments, 50 microvolts criterion threshold ERGs were recorded at minute intervals during the course of 35 minutes of dark adaptation. At the end of this period, the intensity of the stimulus was increased in 0.5 log. unit increments up to full available intensity. Paired flashes at one second interval were used. Five experiments were made.

In the second series, the same light pre-adaptation and dark adaptation technique were used. At the end of the dark adaptation, stimulus intensity was also increased in $0.5 \mathrm{log}$. unit increments up to full intensity. Then, a faint background was projected in the Ganzfeld sphere using a $4 \mathrm{log}$. unit of neutral density filter in front of the background source and again intensities were increased by $0.5 \mathrm{log}$. unit increments. The same procedure was followed using 3.0 and $2.0 \mathrm{log}$. units of background. Six full series were studied.

\section{Technical data:}

The stimulator used a 300 watts Sylvania Concentrated Arc Lamp Type K 300 light source colimated to a Uniblitz shutter, with a time duration of 100 milli-seconds. Neutral density filters were mounted on discs electromechanically controlled through driven Slow Syn Motors. One disc supported neutral density wratten filters, calibrated from 1 to $9 \log$. units and a second disc, filters from 0.1 to $0.9 \mathrm{log}$. units. The same possibilities existed for the background source, a DYS 600 watts Sylvania Tungsten Lamp. Light from the background and the stimulator source were focused each in a $1 / 4$ inch fiber optic light guide which divides into two $1 / 8$ inch fiber bundles. These four sources were directed on a flat back plate mounted 
against the opening of a ping pong ball, one third of which had been excised. This produced a reflected light into the diffusing sphere. The back plate contained a $1 \mathrm{~cm}$. hole. The light sources were not directly visible to the eye under evaluation. The full intensity of the stimulus was evaluated at 1.8 lumens sec. $/ \mathbf{p i}^{2}$, and that of the background at 2.1 lumens sec./pi².

Responses were pre-amplified $1000 \mathrm{x}$ on Tektronix low-level pre-amplifiers type RM 122, using a pass band of 0.8 to $50 \mathrm{cycle} / \mathrm{sec}$. They were amplified and

\section{MICROVOLTS}

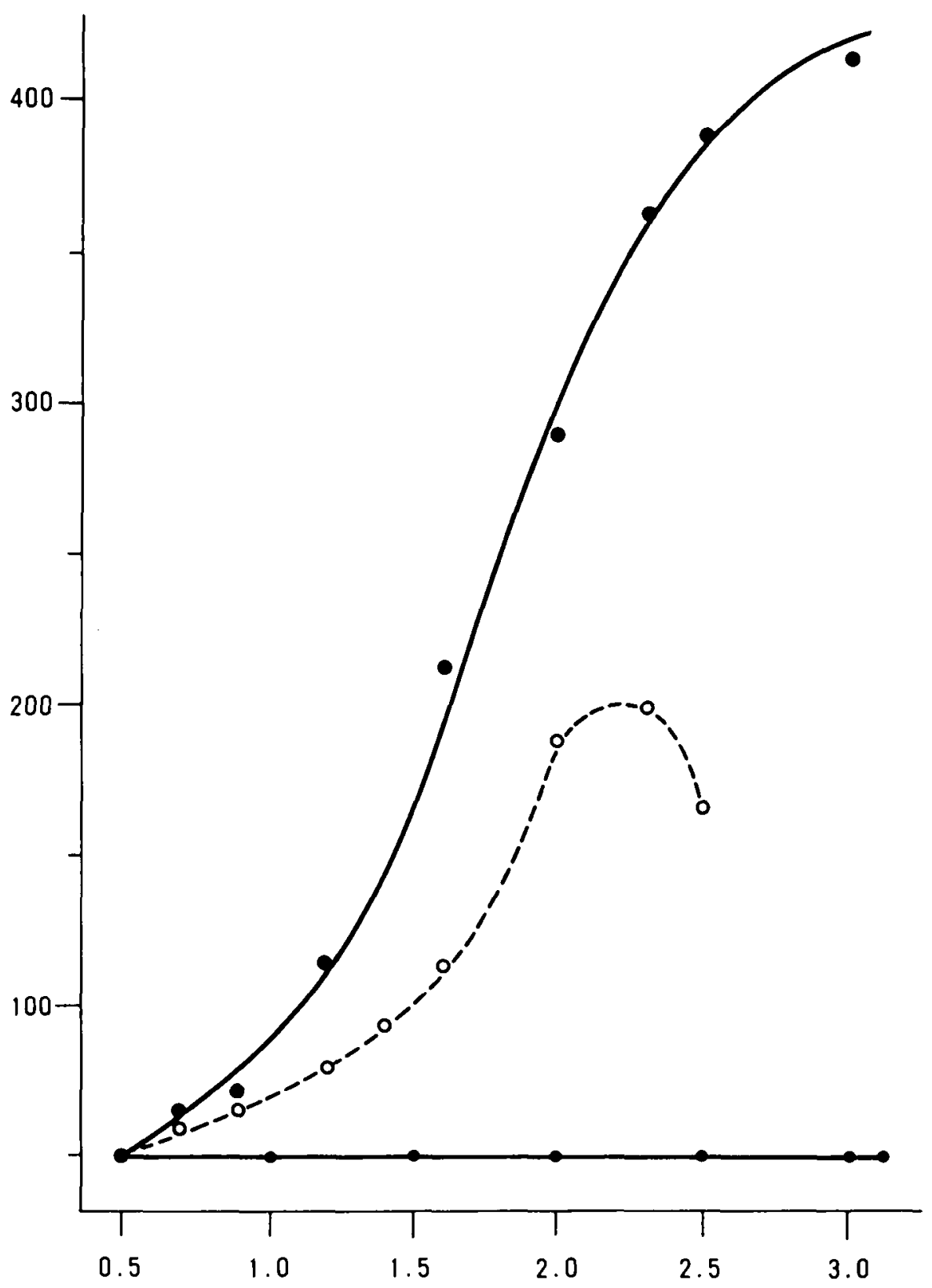

LOG. INTENSITY

Figure 1-Amplitude values in microvolts of rod ERGs recorded in three different states of adaptation. Lowest curve: 50 microvolts criterion threshold response during the course of dark adaptation over a 45 minutes period of time. Highest curve: responses to increasing intensities of stimulation in $0.5 \mathrm{log}$. units increments in a fully dark adapted monkey. Middle curve: same conditions as the preceeding, but second of paired flashes and consequently varying temporary bleaches. Implicit time values of the same ERGs are given in Figure 2.

monitored on a Tektronix type $3 \mathrm{~A} 74$, model 564, four trace oscilloscope and photographed for reproduction. Animals were maintained in a head holder in a shielded dark cage under general anesthesia using a mixture of Halothane $0.5 \%$, oxygen and nitrous oxide. Pupils were maximally dilated.

\section{RESULTS}

Figure 1 presents ERGs obtained in 3 different states of adaptation. The amplitudes of the ERGs have been recorded and measured in 3 conditions. First, during the course of dark adaptation, at a constant criterion response of 50 microvolts. Amplitude was kept constant and is represented by a straight line (lowest in figure 1) and is plotted against stimulation intensity. These intensities were determined by the state of adaptation or sensitivity of the retina.

At the end of the dark adaptation period, the intensity of stimulation was increased by $0.5 \mathrm{log}$. unit increments and the amplitude increased (upper curve of figure 1). Paired flashes were used at one second intervals in the third experiment. Each pair was presented at greater than one minute intervals so as not to cumulatively decrease the state of adaptation. As intensity increased, the resulting temporary bleach decreased the sensitivity and the amplitude of responses to the second flash was correspondingly decreased (middle curve of figure 1) until it became unrecordable.

Points on the three curves of figure 1 corresponding to identical intensities of stimulation were determined and the peak time of the responses in the three conditions was measured. These are shown in figure 2 . The resulting compilation suggests that, whatever the state of adaptation, peak times appear constant for a given stimulation intensity.

Experiments were undertaken to verify these results. Monkeys were fully dark adapted and the stimulation intensity increased in $0.5 \mathrm{log}$. unit increments. Adaptation was then modified by increasing the background by $0.1 \mathrm{log}$. unit intensity increments in steps and recording the responses at each level at vary- 


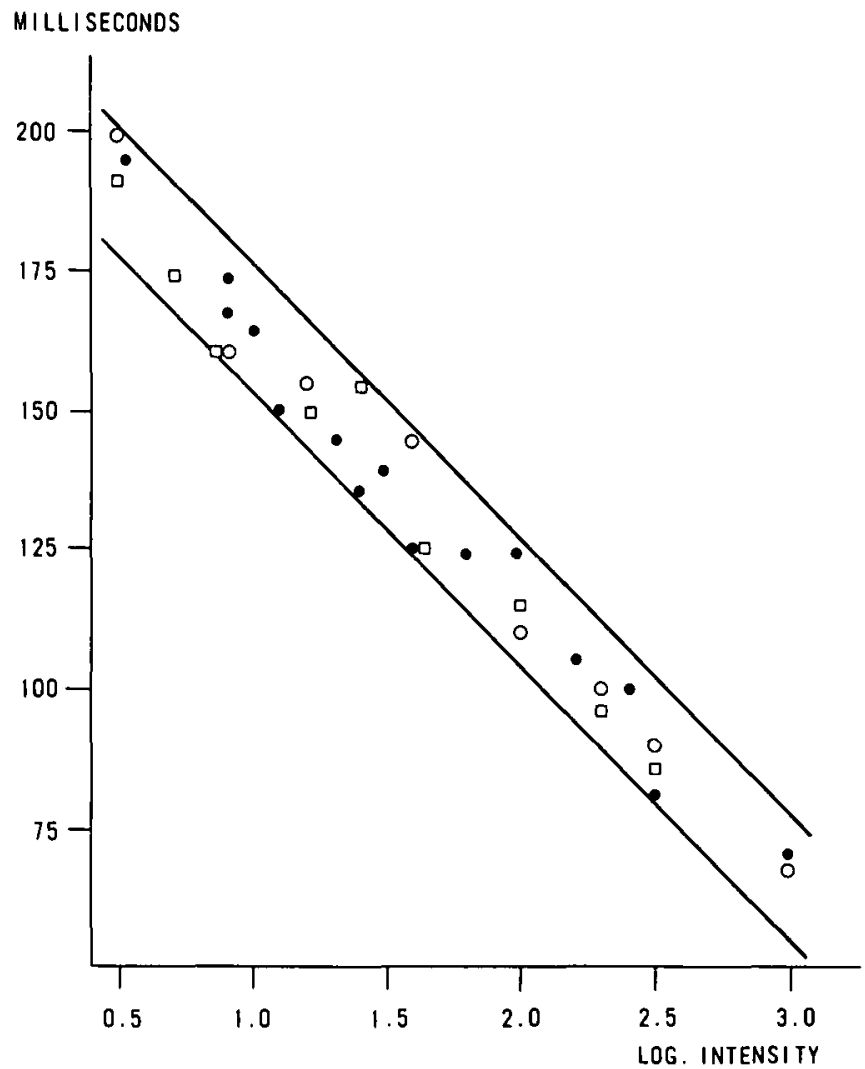

Figure 2-Implicit times of the rod ERG responses the amplitudes of which are presented in figure 1. Peak times appear relatively constant for a given intensity of stimulation in the presence of varying intensities of stimulation. Solid circles: 50 microvolts constant amplitude; open circles: incremental increase of stimulation intensity; squares: same but paired second flash.

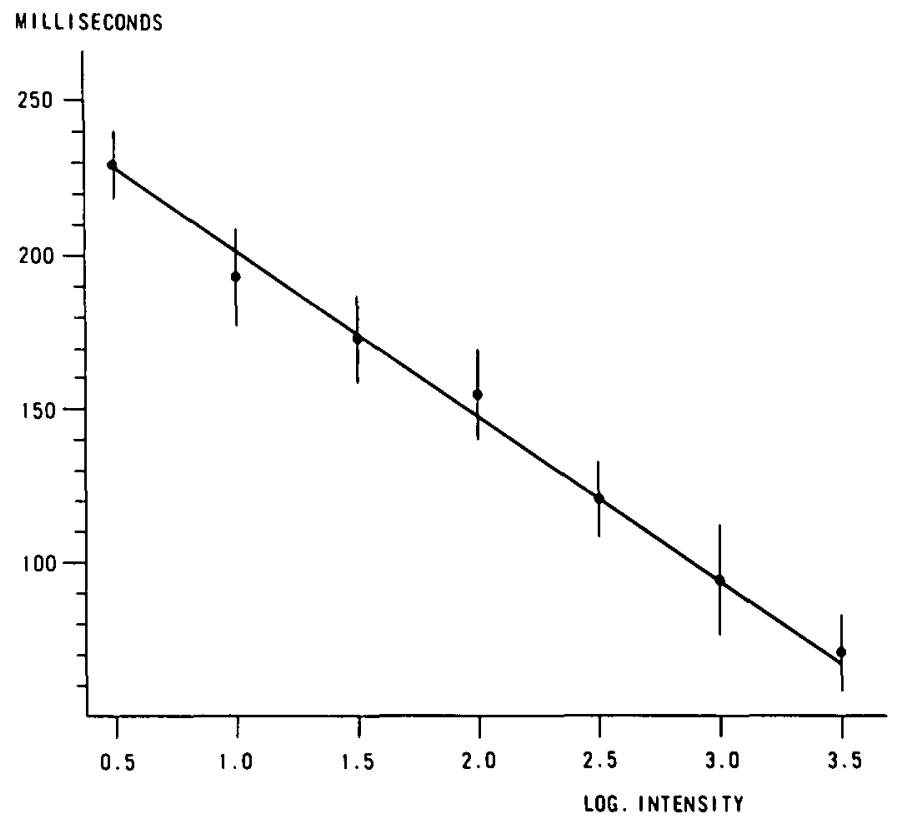

Figure 3-Mean and standard deviations of implicit times of rod ERG responses in six monkeys under full dark adaptation and in the presence of four different backgrounds intensities ( 1 to 4 log. units), for varying intensities of stimulation. All peak times appear constant for any given intensity of stimulation and independent of the state of adaptation. ing intensities of stimulation, again in $0.5 \mathrm{log}$. unit increments. Peak time of each response was measured and the mean value and standard deviation evaluated for each intensity of stimulation. Results are shown in figure 3 . It appears that the peak time of the response is constant for any given intensity. Only rod late responses were used in this computation.

\section{DISCUSSION}

It should be emphasized that these results are based exclusively on the responses of one type of receptors, rods. Cones have much less time variation, if any. This is so during dark adaptation and to variations of intensity of stimulation.

The present observations suggest that the amplitude of the ERG response varies according to the state of adaptation or sensitivity of the retina for a given intensity of stimulation. For example (see fig. 1), amplitudes for $2.5 \mathrm{log}$. units of stimulation are respectively of 50,165 and 410 microvolts, depending on the state of adaptation. However, at this stimulation intensity, peak times of the response are identical as shown in figure 2, where all three peak times are approximately 85 milliseconds at the same intensity of 2.5 log. unit.

If these results are valid, it can be argued that the electrophysiolgical response of the retinal network simultaneously signals first, the state of adaptation and second, the absolute value of the intensity of the stimulus. The first is manifest by the amplitude of the response while the second is dependent on the peak time of the response.

We think that regardless of the amount of photo pigments present or the amount destroyed by bleach, when light strikes, its intensity will produce a constant biochemical or physical effect which determines the speed of the consequent electrical phenomena in the receptor system, as long as the photo-receptor is responsive. Similar results have been found by studying light induced resistance of retinal rods and cones (Lasansky and Marchiafava, 1974). Whether the state of adaptation is dependent on the concentration of pigment, the rate of regeneration or a more proximal electrical feedback, it will control the response or manifest its state through amplitude modulation.

This observation has a practical point in clinical ERG interpretation. When responses are delayed, as in tapeto-retinal degenerations, it is easy to evaluate this delay. However, low intensities of stimulation, 
such as 50 microvolt threshold, produce a different situation. When the threshold value is normal but peak time delayed, the delay can be measured from the normal expected peak time for threshold. However, when threshold is elevated and peak time delayed, should the delay be measured from the normal threshold peak time value or from the estimated peak time for the intensity needed to elicit this threshold response? Lesions of the retina, which destroy parts of the retina leaving the rest of it normal or functional, produce results different from tapeto-retinal degenerations.
Threshold is elevated but the time of response is that which would be expected for that intensity in a normal, although the response is of threshold amplitude.

It is suggested that peak time values in clinical ERG should be evaluated by measuring the pathological peak time from the normal peak time at the given intensity used to obtain the ERG. This conclusion stems from the observations that the peak time of the response is directly related to the intensity of the stimulus while amplitude is modulated by the sensitivity of the retina. This appears to be true for rods within usual clinical experimental conditions.

\section{REFERENCES}

BERSON, E. L., GOURAS, P., HOFF, M. (1969). Temporal aspects of the electroretinogram. Arch. Ophth. 81, 207-214.

BRUNETTE, J. R. (1973). A standardizable method for separating rod and cone responses in clinical electroretinography. Am. J. Ophth. 75, 833-845.

BRUNETTE, J. R., LAFOND, G. (1977). Intensity of stimulation and rods and cones evaluation in clinical ERG. Canad. J. Ophthal. 13, 27-30.

LASANSKY, A., MARCHIAFAVA, P. L. (1974). Light induced resistance changes in retinal rods and cones of the tiger salamander. J. Physiol., 236, 171-191. 